\title{
DEVELOPMENT OF CROSS-ROLLING TEXTURES IN ARMCO-IRON
}

\author{
A. BÖCKER, H. KLEIN and H. J. BUNGE \\ Department of Physical Metallurgy, TU Clausthal, FRG
}

(Received May 18, 1989)

\begin{abstract}
ARMCO-iron was cross-rolled in two different modes, i.e. with a rotation of the rolling direction through $90^{\circ}$ after each individual pass or after half of the reduction in thickness only. In the first case, which corresponds to a truly tetragonal straight deformation path, the orientation $\{001\}\langle 110\rangle$ assumes an extremely high orientation density of $50 \times$ random after $93 \%$ reduction. Besides this, the texture contains a nearly ideal $\langle 111\rangle \| N D$-orientation tube, the intensity of which increases to about $10 \times$ random. The texture of the second mode is similar to that of the first one up to about $50 \%$ reduction. After that the density in the orientation $\{001\}\langle 110\rangle$ decreases and increases again at the highest deformation degrees. Two minor orientation components $\{001\}\langle 100\rangle$ and $\{011\}\langle 100\rangle$ were found with intensities in the range of 3 to $5 \times$ random at low deformation degrees of about $10 \%$.
\end{abstract}

KEY WORDS Cross-rolling, texture evolution, ARMCO-iron.

\section{INTRODUCTION}

The rolling texture of iron and other bcc metals has often been described by three ideal orientations $\{001\}\langle 110\rangle,\{112\}\langle 110\rangle$ and $\{111\}\langle 112\rangle$ with spread ranges between them (see e.g. Haessner and Weik 1956, Kern, Lee, Bunge 1986, see also Wassermann and Grewen 1969). One of the spread ranges is extended between $\{001\}\langle 110\rangle$ and $\{112\}\langle 110\rangle$. It is thus a "limited" $\langle 110\rangle$ fibre texture with the $\langle 110\rangle$-fibre axis parallel to the rolling direction. The other spread range is extended between $\{112\}\langle 110\rangle$ and $\{111\}\langle 112\rangle$. It can be described by a "curved" skeleton line in the orientation space with a variation of the intensity along this line.

Both spread ranges comprise also the orientation $\{111\}\langle 110\rangle$ (Kern, Lee, Bunge 1986). Hence, the rolling texture of iron has been described alternatively by two fibre textures, a "limited" $\langle 110\rangle \|$ RD fibre and a "complete" $\langle 111\rangle \mid N D$ fibre. In this description the ideal orientations of the first description are the intersections of the two "fibres." The two descriptions are thus somehow complementary to each other.

The rolling deformation can be idealized by plane strain deformation, i.e. an elongation in rolling direction and a shortening of the same amount in normal direction, whereas the transverse direction remains constant. This ideal shape change may especially be assumed to be valid in the central layer of a rolled sheet. Hence, the texture development by rolling is usually studied in samples taken from the central layer of the sheet. Plane strain deformation possesses orthorhombic symmetry, i.e. rolling direction, transverse direction and normal direction are axes of two-fold symmetry. 
If the rolling direction is being turned through $90^{\circ}$ after each rolling pass, i.e. the material is being "cross-rolled" then the sample symmetry is tetragonal with a four-fold axis parallel to normal direction instead of the two-fold axis of straight rolling. Also cross-rolling textures of iron and other bcc metals have been studied in detail. A summary of these investigations was given by Wassermann and Grewen in 1969.

The cross-rolling process is truly tetragonal, if a large number of very small rolling passes are used. From the technological point of view, this is, however, difficult to achieve. It is easier to change the rolling direction only once. In this pseudo-cross-rolling process several passes are being made in one direction (with or without reversion). Then the rolling direction is changed by $90^{\circ}$ and again several passes are carried out in this new direction (with or without reversion). Even if the total rolling degrees in both directions are equal, this two-stage rolling does not strictly correspond to tetragonal symmetry. This follows from the fact, that two crystal rotations carried out one after the other lead to different resulting rotations, when their sequence is changed. (Mathematically speaking rotations are not commutative). The earlier investigations of the cross-rolling texture of iron (as reviewed for instance by Wassermann and Grewen 1969) were carried out in terms of pole figures which were then interpreted qualitatively in terms of ideal orientations and spread ranges or fibre axis components. In the case of straight rolling it turned out that the calculation of ODF from pole figures gave much more detailed results since the ODF avoids the superposition of spread ranges encountered in the pole figures. It was thus the purpose of the present

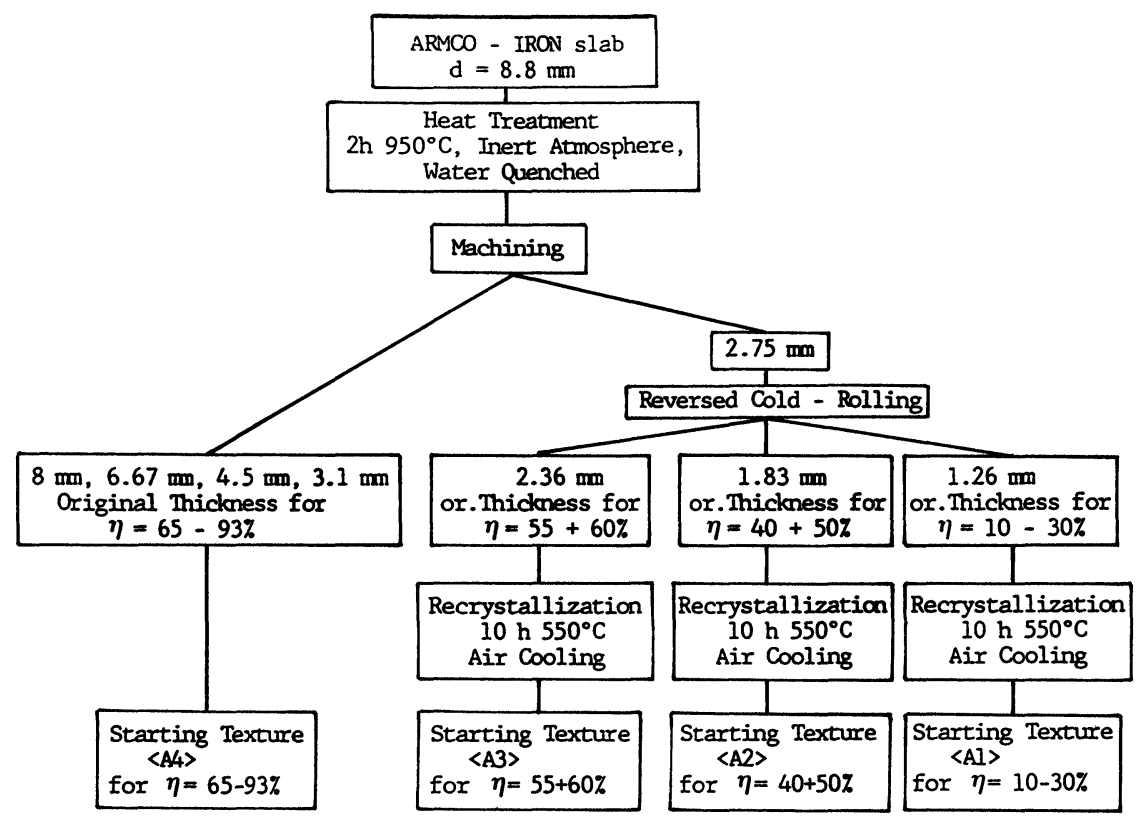

Figure 1 Pre-treatment of ARMCO-iron slabs before cold rolling. 


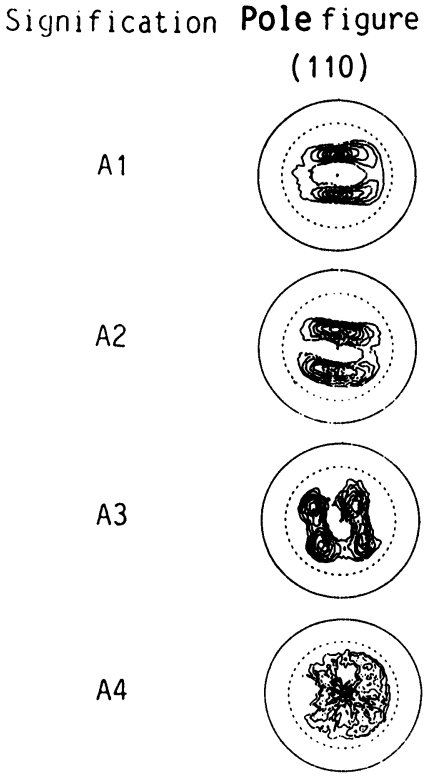

(a)
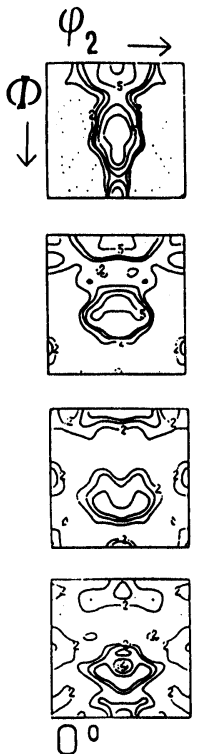
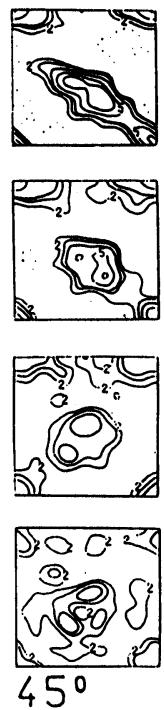

(b)
$I_{\max }$

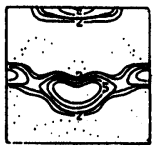

5.74

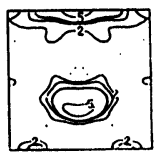

4.15
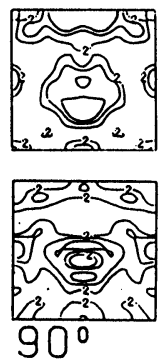

3.73

(c)

Figure 2 Textures of the initial states A1 to A4. a) (110) pole figues b) three ODF sections c) Maximum orientation density.

paper to study texture development during cross-rolling in ARMCO-iron using the ODF representation. Similar studies were recently published by Öztürk (1988) for cross-rolled fcc metals copper and brass.

\section{EXPERIMENTAL PROCEDURE}

The material studied was ARMCO-iron with $0.03 \% \mathrm{C}$ which was received in the form of hot rolled sheet $8.8 \mathrm{~mm}$ thick. It was at first heat treated for $2^{h}$ at $950^{\circ} \mathrm{C}$ in an inert atmosphere and water quenched. In order to obtain similar final thicknesses after various degrees of cross-rolling, the initial size of the sheets was reduced by machining and reversed cold rolling followed by recrystallization as shown in Figure 1 . The grain structures of the initial states A1 to A3 were slightly elongated in rolling direction whereas the grains of the material A4 were nearly equiaxed, the grain size was in the order of magnitude of $67 \mu \mathrm{m}$. The textures of the initial states were not random as is shown in Figure 2. The maximum orientation density values also given in Figure 2 show that these textures were of medium degree. Cross-rolling was carried out on a two-roll laboratory mill with $180 \mathrm{~mm}$ roll diameter. Slabs of $60 \times 60 \mathrm{~mm}$ were cut from the pre-treated material. The slabs were then cross-rolled in two different ways: 


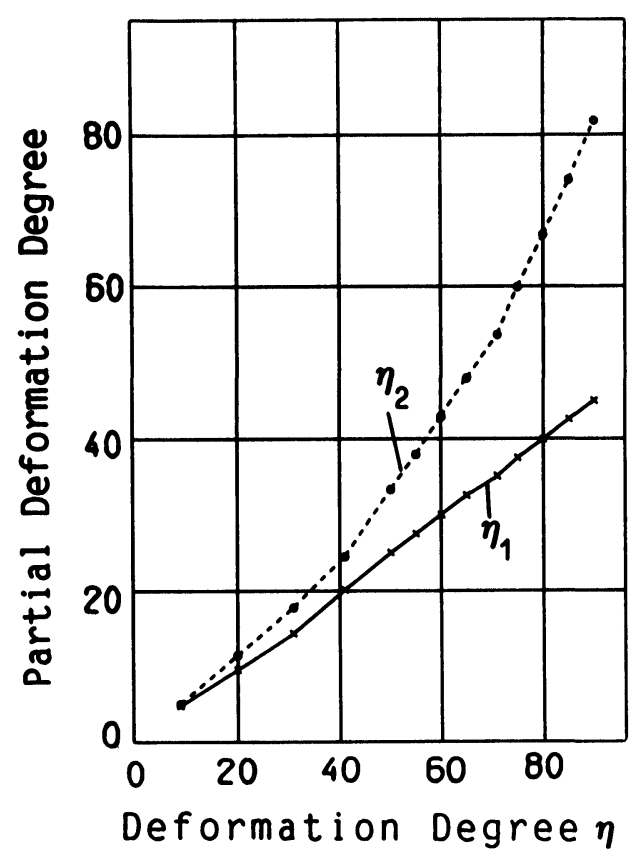

Figure 3 The two partial deformation degrees for two-stage rolling.

\section{Two-Stage Rolling}

The slabs were rolled in the original rolling direction to about half the final reduction in thickness by reversing after each pass. After that they were rolled in the original transverse direction also reversing after each pass. Thereby the two partial deformation degrees $\eta_{1}$ and $\eta_{2}$ are different as is shown in Figure 3.

\section{Multi-Stage Rolling}

In this case the slab was rotated through 90 degrees after each pass up to the final degree. The nominal and actual deformation degrees, the number of passes and the reduction per pass are given in Table 1 . In order to measure the textures, samples of $33.9 \mathrm{~mm}$ diameter were taken from all rolled sheets. They were ground with $\mathrm{SiC}$ paper to about the middle of the sheet and then etched with an etchant of $45 \mathrm{ml} \mathrm{C}_{2} \mathrm{H}_{5} \mathrm{OH}+10 \mathrm{ml} \mathrm{HNO}_{3}$. Hence, the obtained textures correspond to the central layers of the sheets. Texture measurements were carried out with the automatic texture goniometer ATEMA-C using $\mathrm{MoK}_{\alpha}$ radiation. Four incomplete pole figures (110) (200) (211) (311) were measured in the back-reflection range $\alpha \leq 70^{\circ}$. The measurements were done in steps of $\Delta \alpha=5^{\circ}$ and integrating through $\Delta \beta=3.6^{\circ}$. The intensity values were corrected for background scattering. Complete ODF were calculated using the series expansion 
Table 1 Nominal and actual rolling degree, number of passes and mean reduction per pass. a) Two-stage rolling b) Multi-stage rolling

\begin{tabular}{lccl}
\hline $\begin{array}{l}\text { Rolling degree } \\
\text { Nominal }\end{array}$ & Actual & $\begin{array}{l}\text { Number } \\
\text { of passes }\end{array}$ & $\begin{array}{l}\text { Mean reduction } \\
\text { per pass }(\mathrm{mm})\end{array}$ \\
\hline$a)$ & & & \\
$10 \%$ & $9 \%$ & 3 & 0.04 \\
$20 \%$ & $20 \%$ & 10 & 0.03 \\
$30 \%$ & $31 \%$ & 13 & 0.03 \\
$40 \%$ & $41 \%$ & 15 & 0.05 \\
$50 \%$ & $50 \%$ & 18 & 0.05 \\
$55 \%$ & $55 \%$ & 19 & 0.07 \\
$60 \%$ & $60 \%$ & 19 & 0.08 \\
$65 \%$ & $65 \%$ & 23 & 0.09 \\
$70 \%$ & $71 \%$ & 26 & 0.09 \\
$75 \%$ & $75 \%$ & 41 & 0.08 \\
$80 \%$ & $80 \%$ & 47 & 0.08 \\
$85 \%$ & $85 \%$ & 66 & 0.09 \\
$90 \%$ & $90 \%$ & 94 & 0.08 \\
$93 \%$ & $93 \%$ & 103 & 0.07 \\
& & & \\
$b)$ & & & \\
$10 \%$ & $10 \%$ & 7 & 0.02 \\
$20 \%$ & $18 \%$ & 12 & 0.02 \\
$30 \%$ & $31 \%$ & 16 & 0.02 \\
$40 \%$ & $42 \%$ & 16 & 0.05 \\
$50 \%$ & $50 \%$ & 20 & 0.05 \\
$55 \%$ & $52 \%$ & 22 & 0.06 \\
$60 \%$ & $59 \%$ & 25 & 0.06 \\
$65 \%$ & $64 \%$ & 35 & 0.06 \\
$70 \%$ & $69 \%$ & 41 & 0.05 \\
$75 \%$ & $74 \%$ & 74 & 0.05 \\
$80 \%$ & $79 \%$ & 78 & 0.05 \\
$85 \%$ & $95 \%$ & 73 & 0.08 \\
$90 \%$ & $90 \%$ & 134 & 0.05 \\
$93 \%$ & 142 & 0.05 \\
\hline & & &
\end{tabular}

method with $\lambda=22$ for the even part. The odd part with $\lambda=21$ was obtained by the zero-range method.

\section{RESULTS}

The initial textures before cross-rolling were already given in Figure 2. The (110) pole figures of the cross-rolled samples are shown in Figure 4. It is seen that they exhibit four-fold symmetry after higher deformation degrees of the multi-stage case. This mode of deformation corresponds to tetragonal symmetry with a four-fold axis in sheet normal direction. The lower deformation degrees of this mode still show remnants of the two-fold symmetry due to the starting textures which had only the orthorhombic sample symmetry.

The textures after two-stage rolling deviate more or less from tetragonal symmetry particularly after the higher deformation degrees. 
Two-Stage Rolling

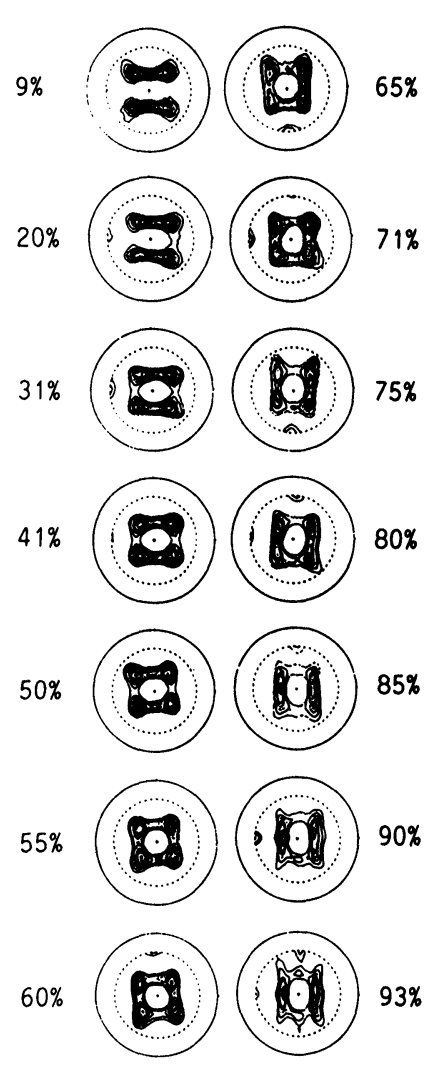

(a)
Multi-Stage Rolling
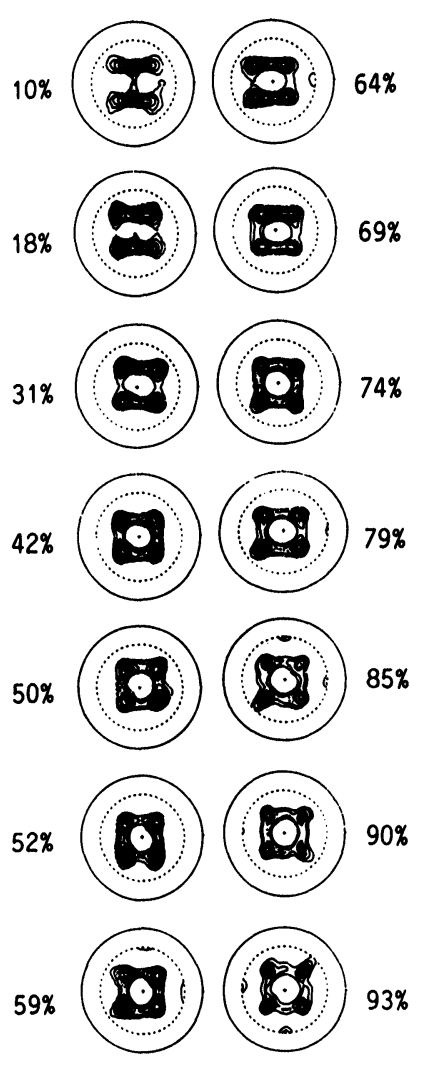

(b)

Figure 4 (110)-pole figures of the rolling degrees $10 \%$ up to 93\%, a) Two-stage rolling b) Multi-stage rolling.

The ODF of three deformation degrees of each deformation mode are shown in Figure 5. The main features of these textures can be described in terms of an orientation tube running through the whole orientation space as well as three ideal orientations and spread ranges between them. This is illustrated schematically in Figure 6 . The orientation tube is extended approximately between the orientations $A=(111)[110]$ and $B=(111)[112]$.

According to Figure 6 the main features of the textures can be seen in the sections $\varphi_{1}=0^{\circ}, 45^{\circ}, 90^{\circ}$, which are thus shown in Figure 7 for all studied textures. In this representation the tetragonal sample symmetry expresses itself in identical density distributions in the sections $\varphi_{1}=0^{\circ}$ and $\varphi_{1}=90^{\circ}$. It is clearly seen that this symmetry is found in the multi-stage mode after high deformation degrees but not in the two-stage mode.

In order to estimate possible series truncation errors, the mean absolute values of the coefficients $C_{L}^{\mu \nu}$ are plotted in Figure 8 as a function of the degree $L$. They 

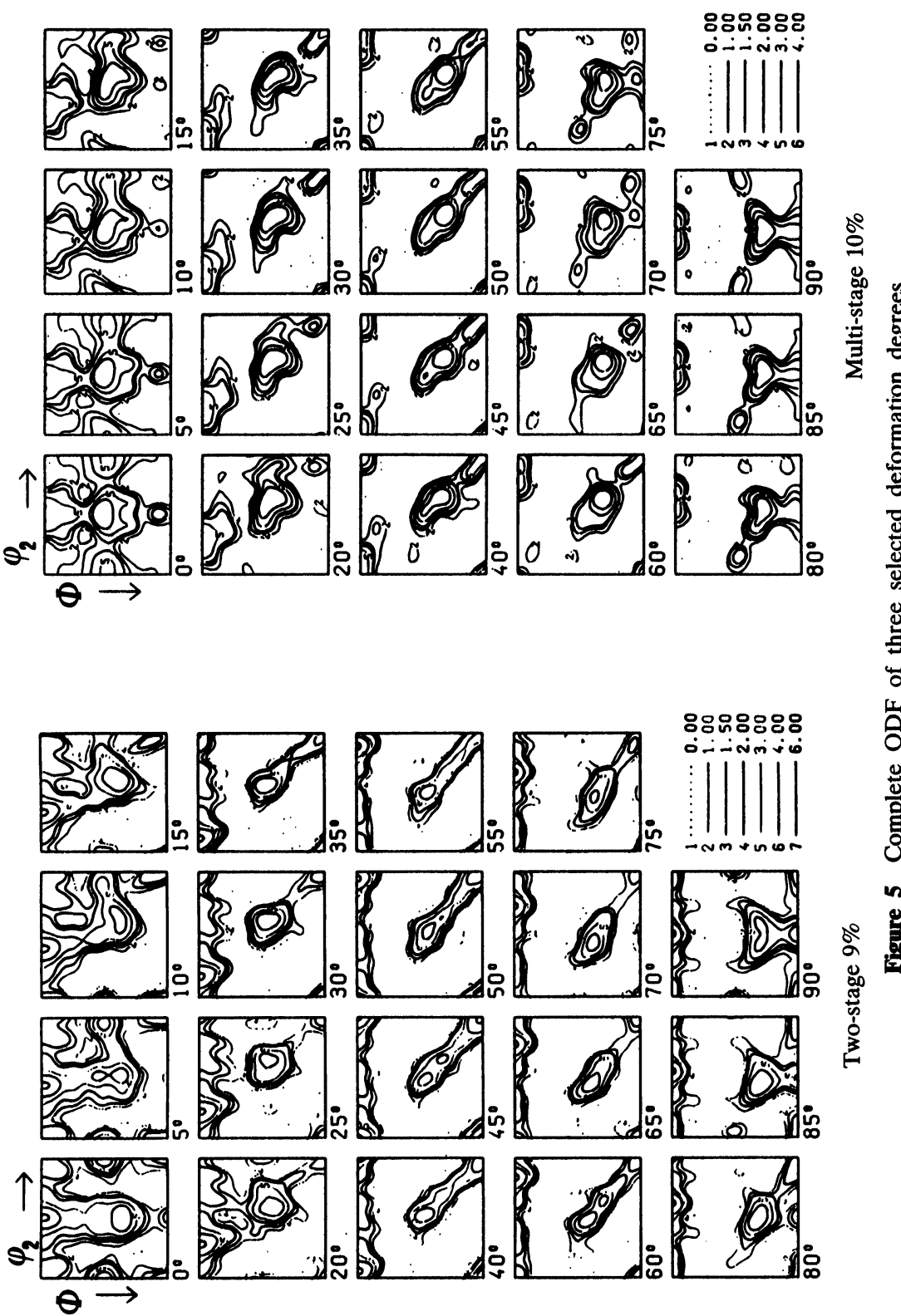

$\theta \rightarrow$ 

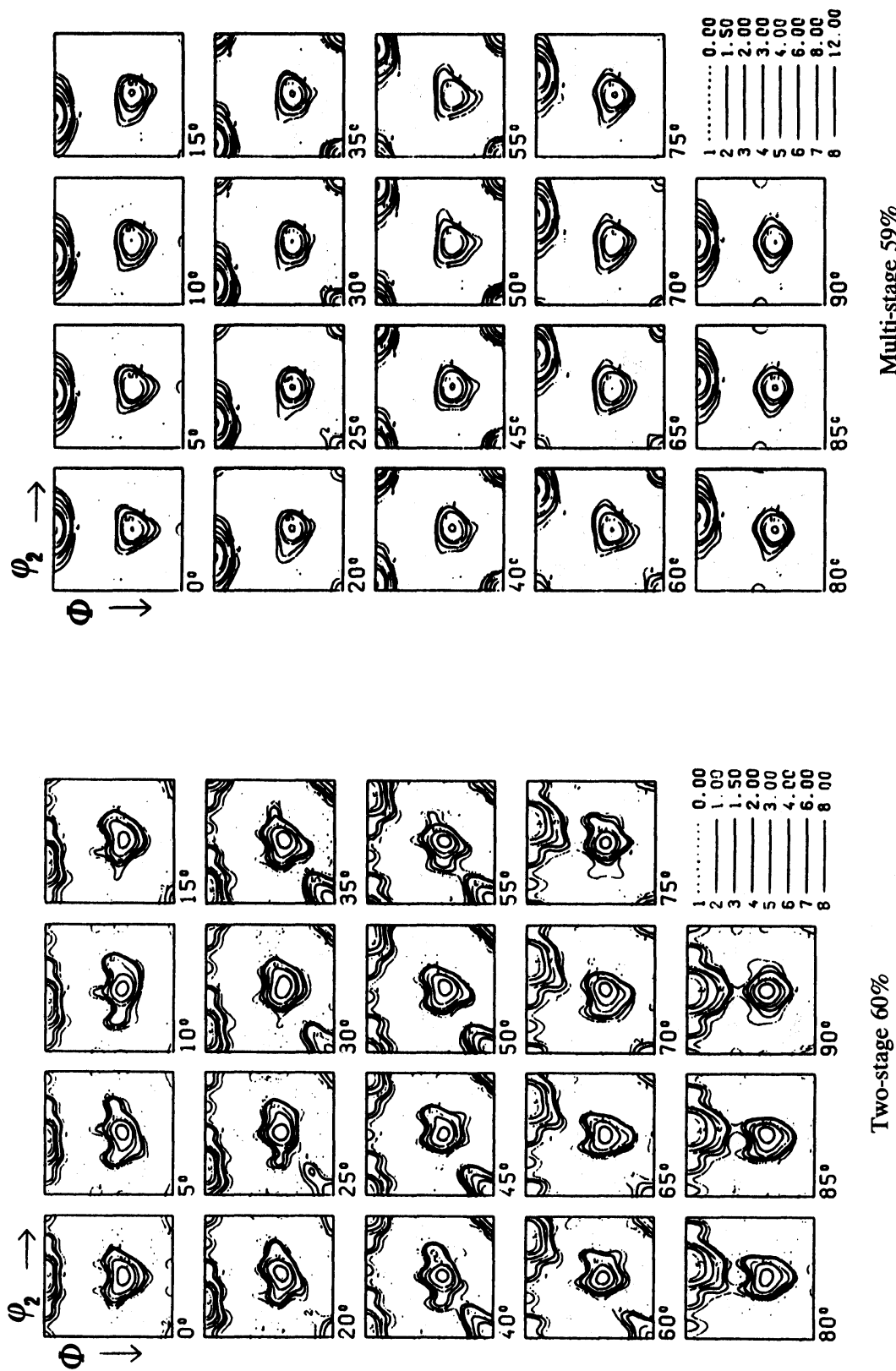

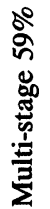




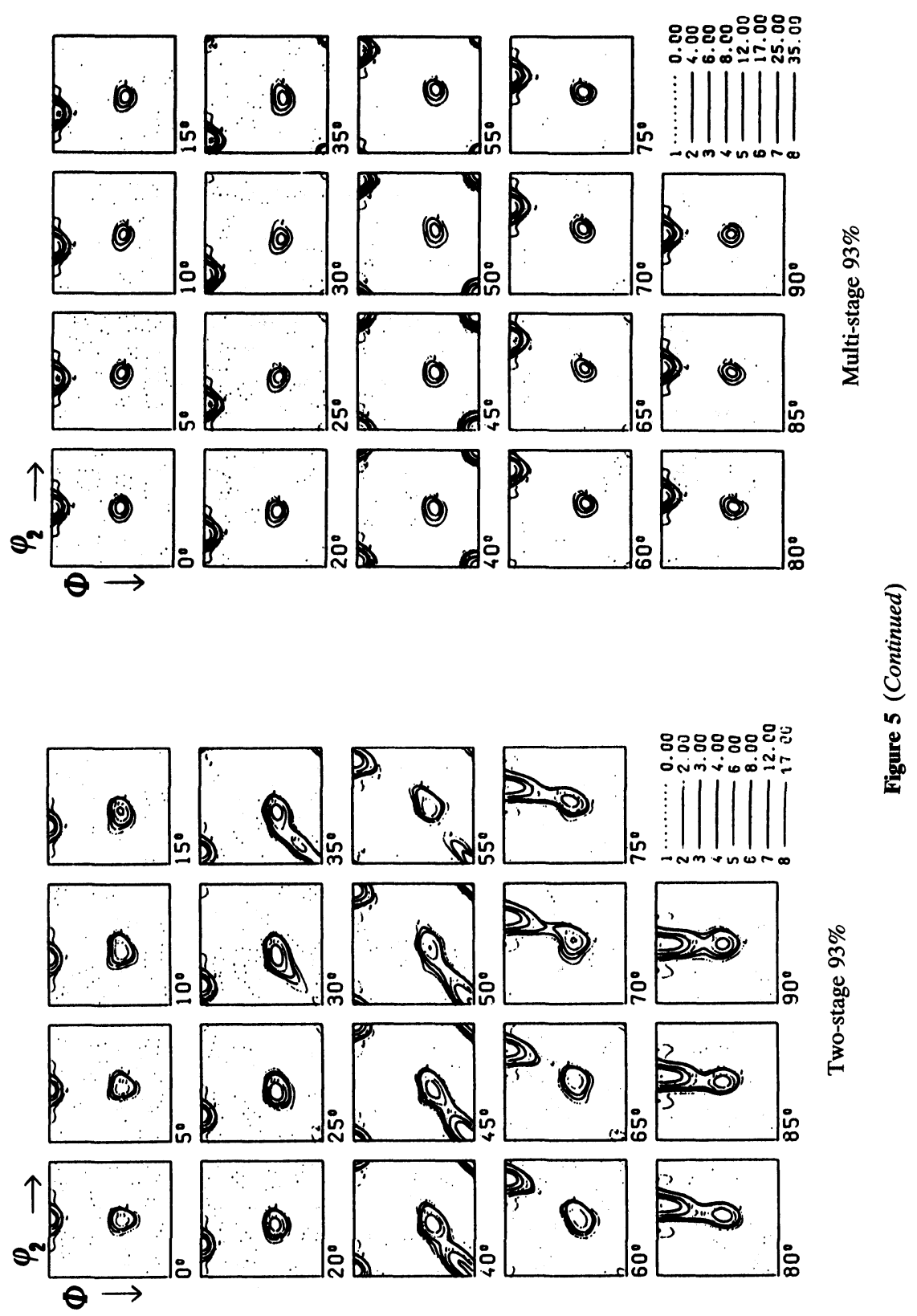



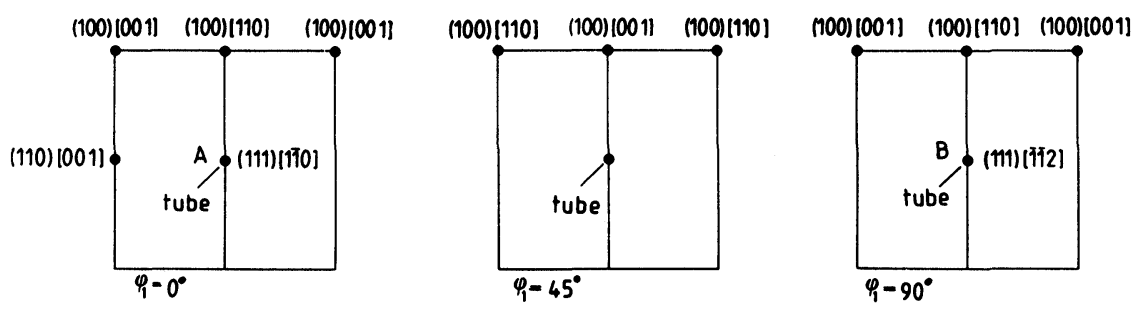

Figure 6 Main orientations of the ODFs of Figure 5.

are compared with the corresponding error quantities obtained from the compatibility conditions of the different pole figures. It is seen that the coefficients have nearly converged to the order of magnitude of their respective errors at $L=22$ for the low and medium deformation degrees, whereas they are a bit larger than the errors for the high degrees. Hence, truncation errors are not expected at low and medium degrees, whereas the ODFs of the highest degrees may be slightly broadened by the series truncation effect.

The four-fold symmetry observed in the pole figures also expresses itself in the
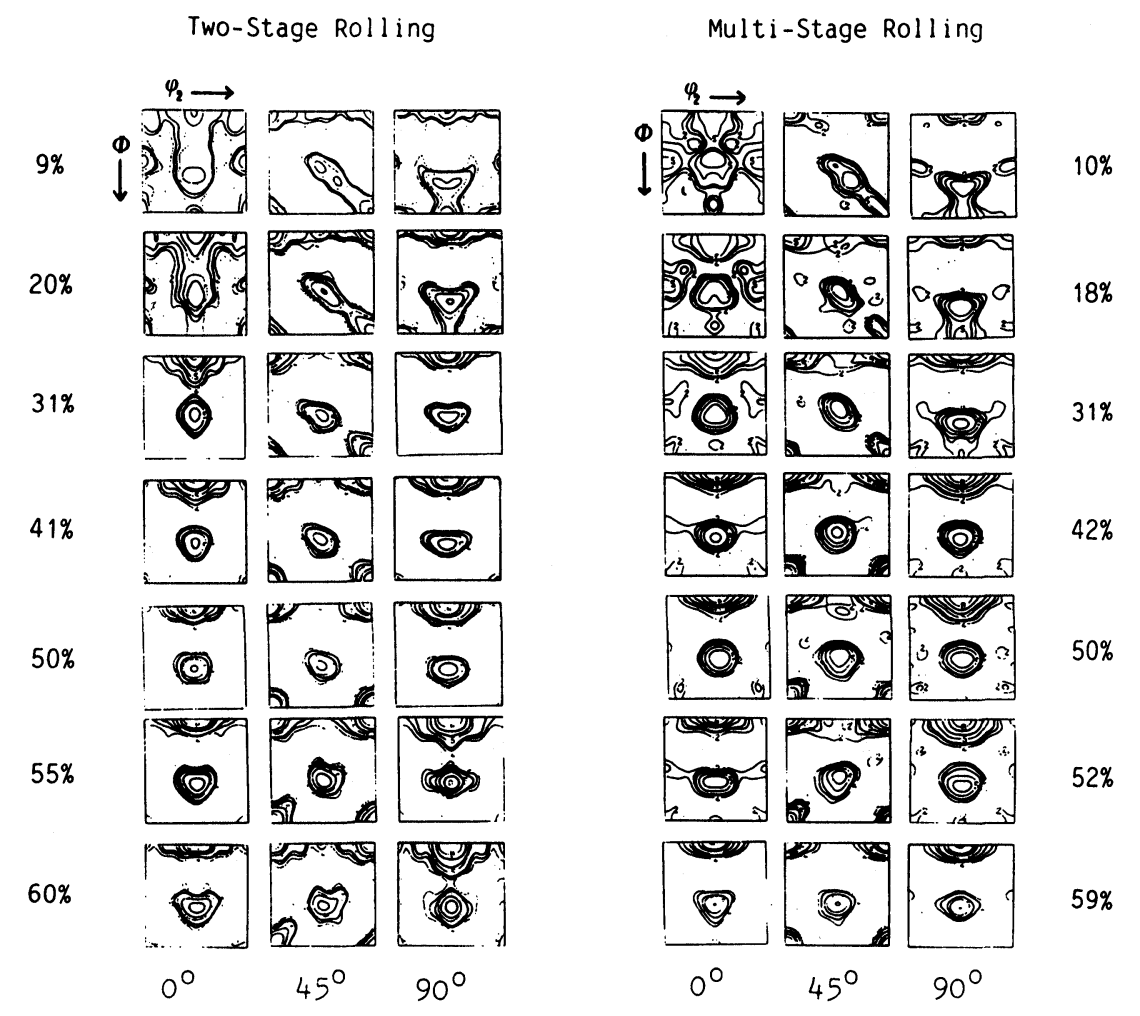

Figure 7 Three sections of the ODFs of cross-rolled ARMCO-iron. 

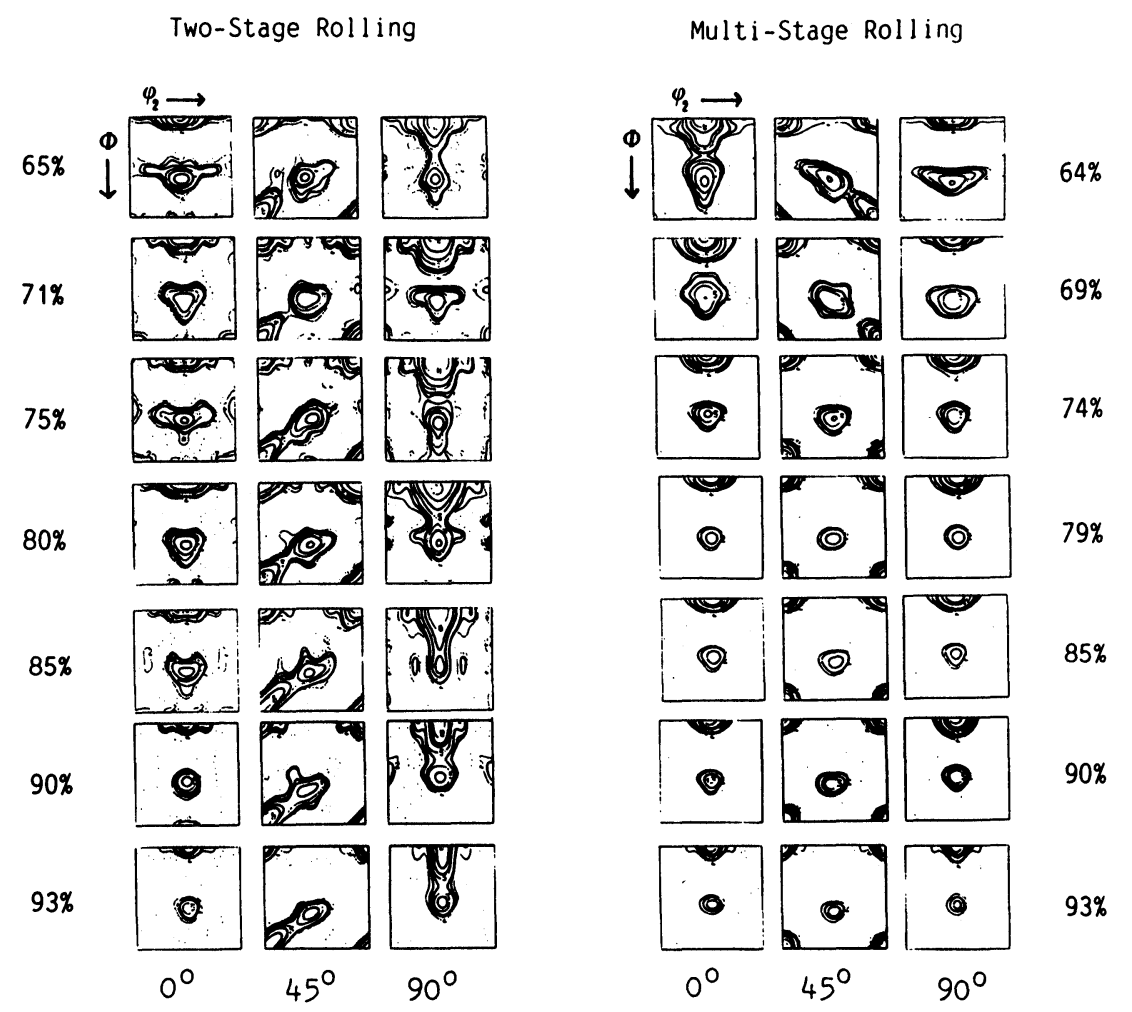

Figure 7 (Continued)

texture coefficients $C_{L}^{\mu \nu}$. This symmetry requires $C_{L}^{\mu v}=0$ for $v \neq 4 n$. Hence, in Figure 9 the mean absolute values of these coefficients are plotted separately for $v=4 n$ and $v \neq 4 n$. It is seen that in the multi-stage mode the textures approach continuously the tetragonal sample symmetry. In the two-stage mode, this symmetry is approximately found after medium deformation degrees whereas it is violated in low and high deformation degrees.

As was already seen, the continuous orientation tube is extended approximately between the orientations $A$ and $B$ shown in Figure 6 . In these orientations the (111) plane is approximately parallel to the rolling plane. The same holds for the whole orientation tube as can be seen in Figure 10 which shows the skeleton line of this tube represented by the orientation of normal direction and rolling direction in the respective inverse pole figures.

In detail, small deviations of the orientations $A$ and $B$ from ideal low-index orientations were found as is seen in Figure 11 which gives the values $\phi$ of these two orientations as a function of the deformation degree. For the ideal position with $\langle 111\rangle$ parallel to normal direction this value should be $\phi=54.7^{\circ}$ as is also indicated in Figure 11.

The orientation density along the skeleton line is shown in Figure 12. It is seen that the density is nearly constant along the skeleton line except for the higher 

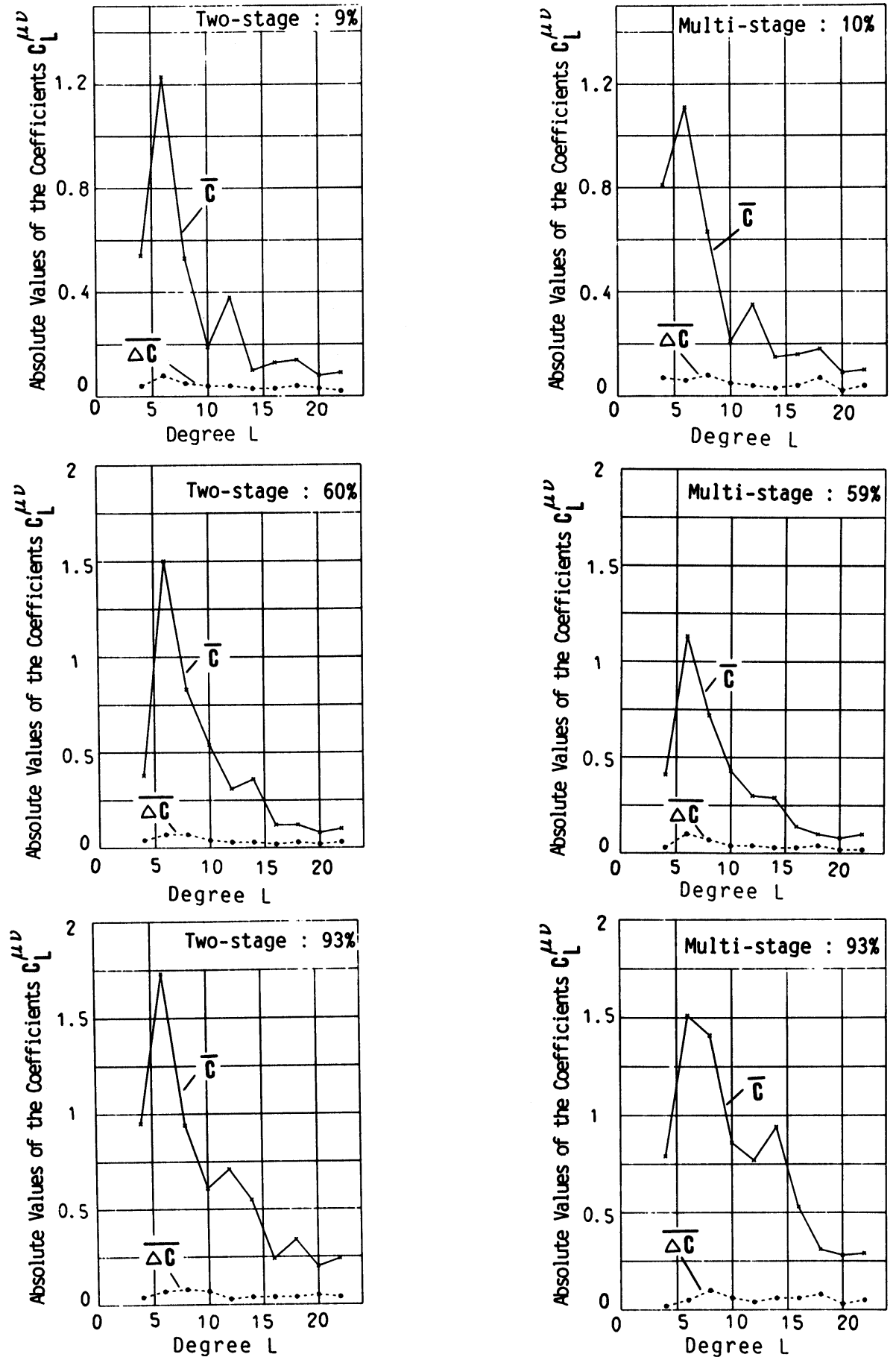

Figure 8 Mean absolute values $\left|\overline{C_{L}^{\mu \nu}}\right|$ of the texture coefficients and the corresponding error quantities $\mid \overline{\Delta C_{L}^{\mu \nu} \mid}$ of the three selected deformation degrees. 

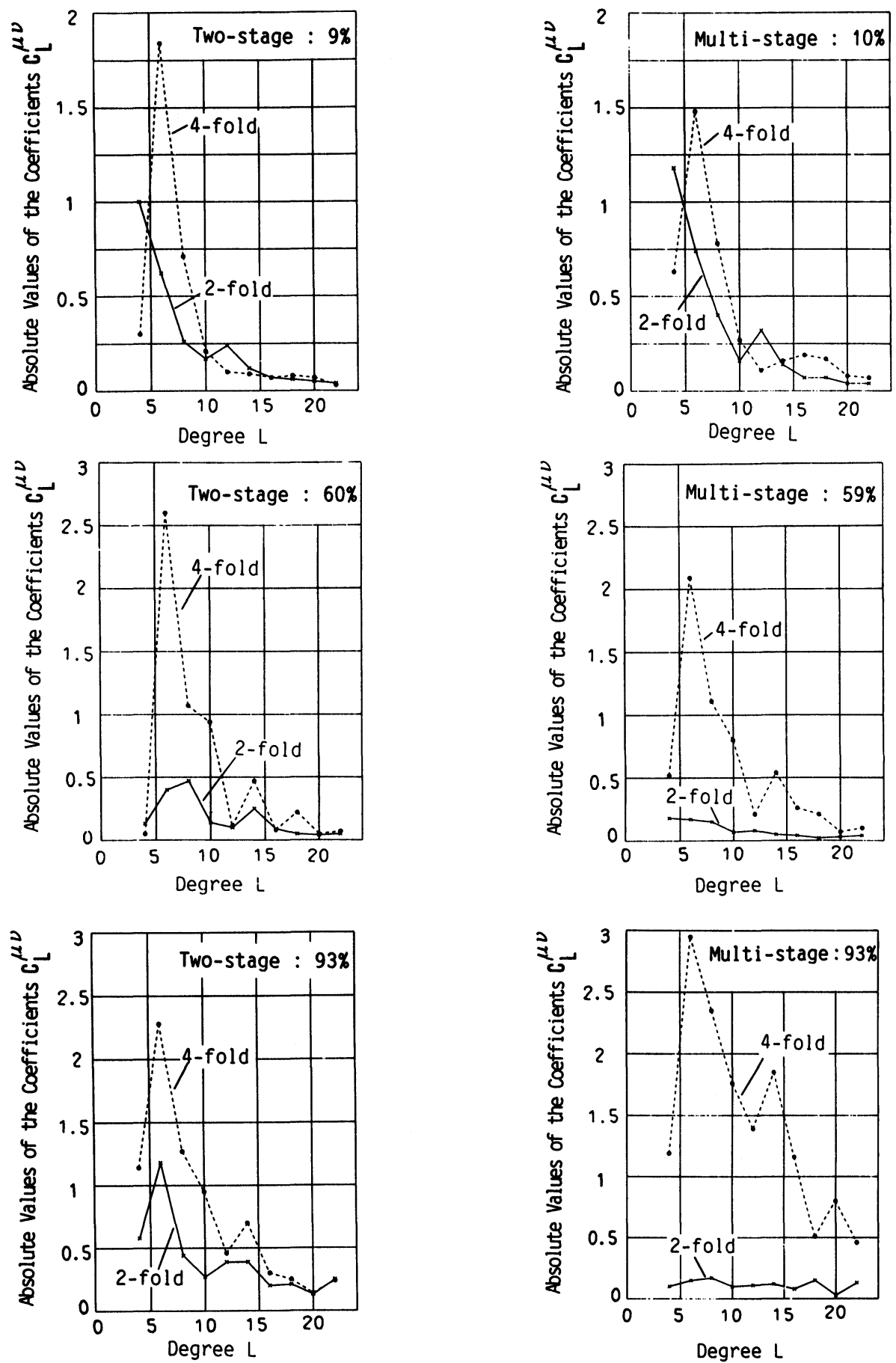

Figure 9 Mean absolute values of the 4-fold and 2-fold texture coefficients $C_{L}^{\mu \nu}$ for three selected deformation degrees. 
A. BÖCKER ET $\boldsymbol{A L}$.

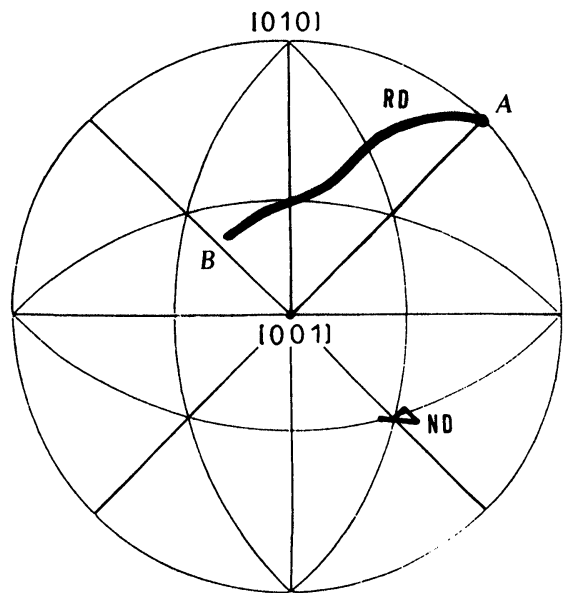

(a)

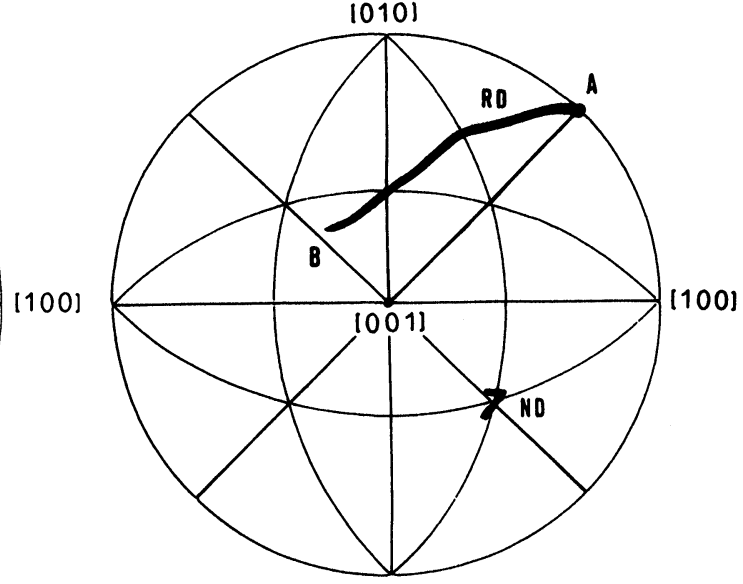

(b)

Figure 10 Skeleton line of the orientation tube represented in the inverse pole figures of rolling and normal direction. (a) Two-stage rolling. (b) Multi-stage rolling.

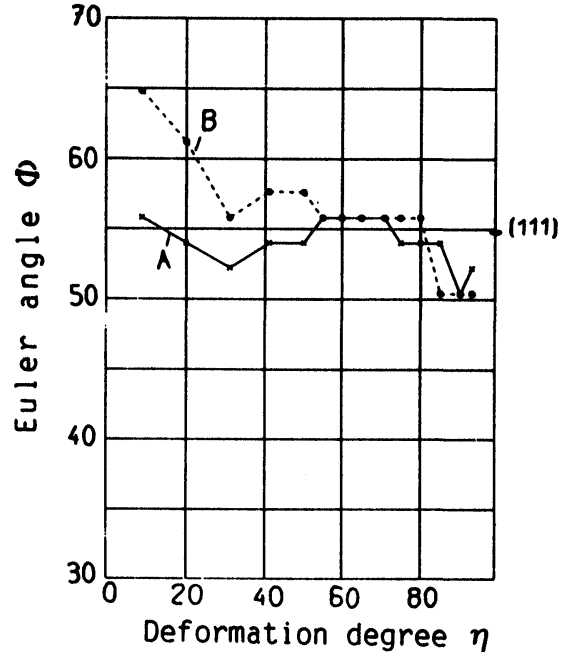

(a)

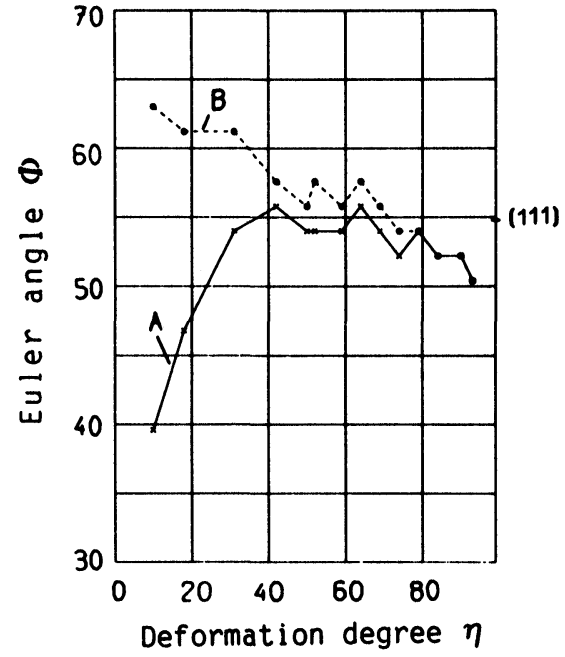

(b)

Figure 11 Exact position of the orientations $A$ and $B$ of Figure 5 described by the angle $\phi$. (a) Two-stage rolling. (b) Multi-stage rolling. 


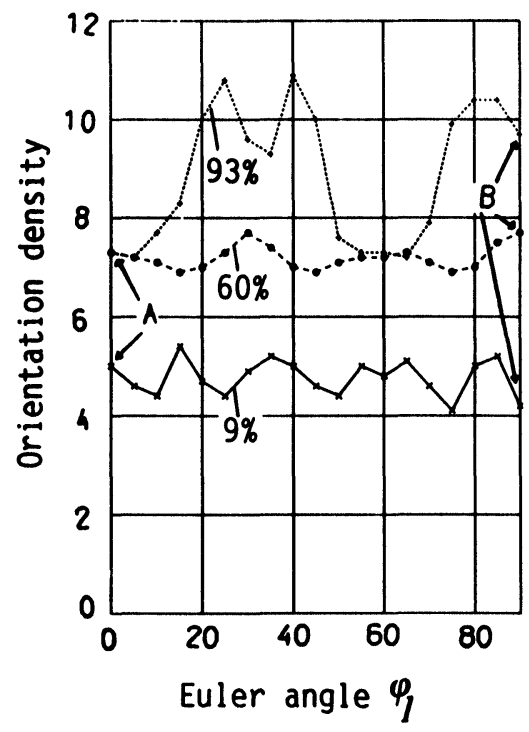

(a)

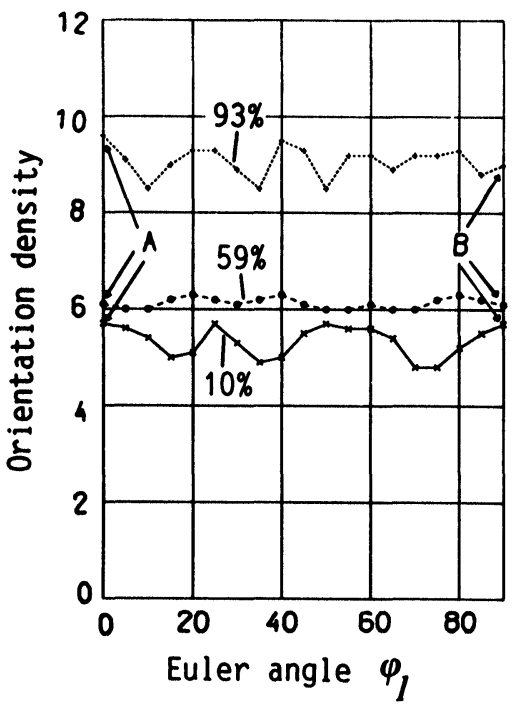

(b)

Figure 12 Orientation density along the skeleton line. (a) Two-stage rolling. (b) Multi-stage rolling.

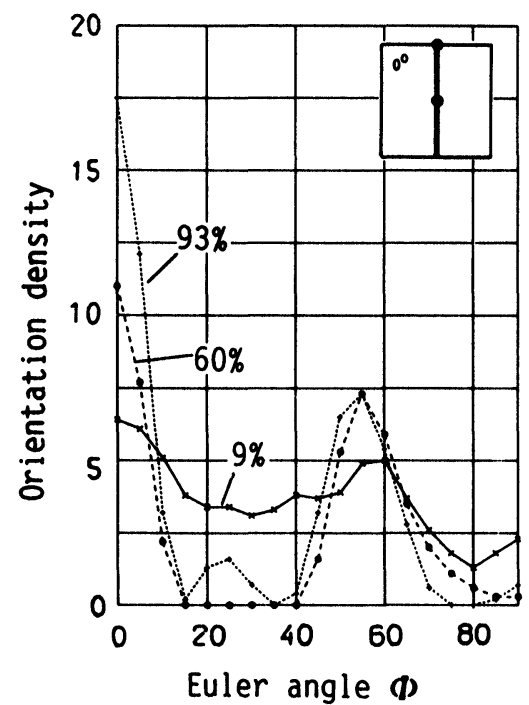

(a)

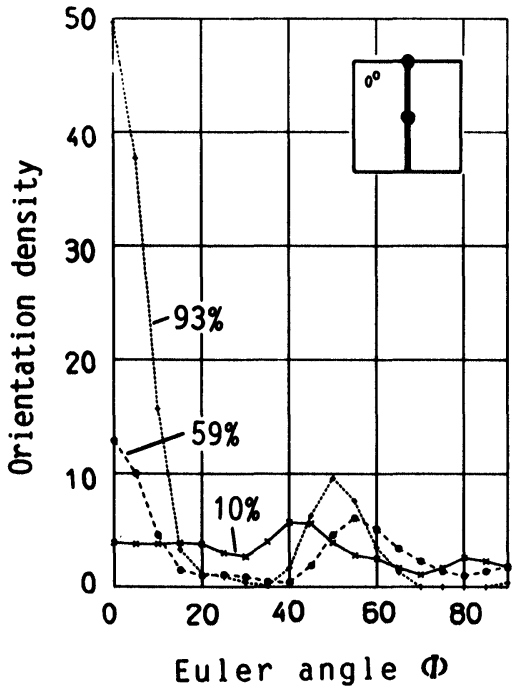

(b)

Figure 13 Orientation density along the section $\varphi_{1}=0^{\circ}, \varphi_{2}=45^{\circ}$. (a) Two-stage rolling. (b) Multi-stage rolling. 


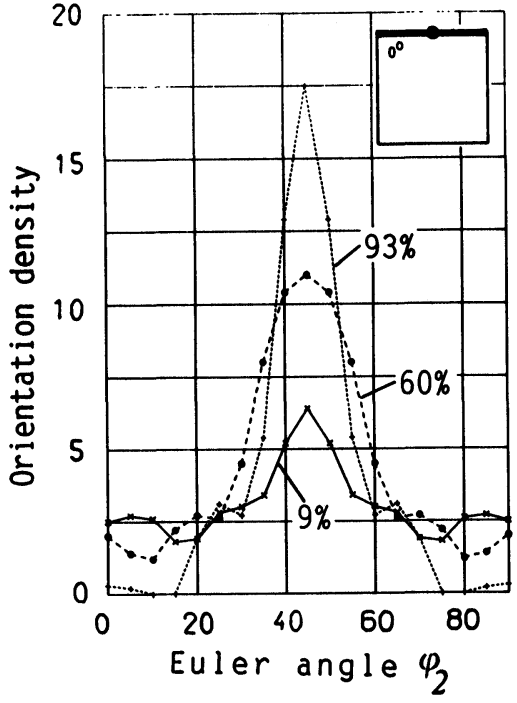

(a)

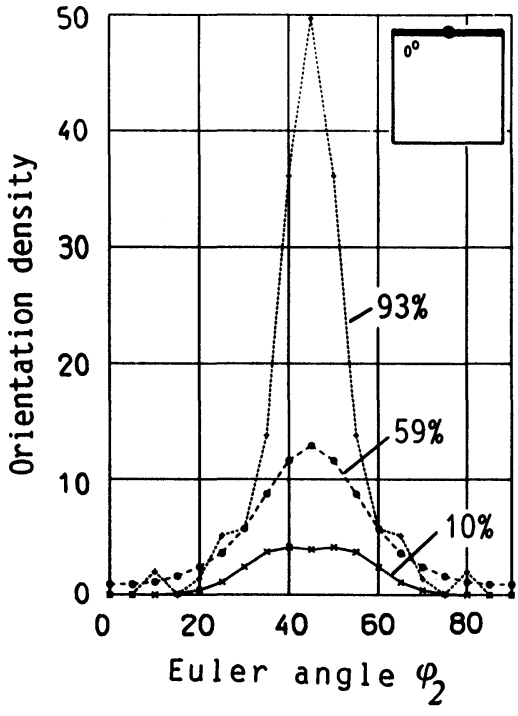

(b)

Figure 14 Orientation density along the section $\varphi_{1}=0^{\circ}, \phi=0^{\circ}$. (a) Two-stage rolling. (b) Multi-stage rolling.

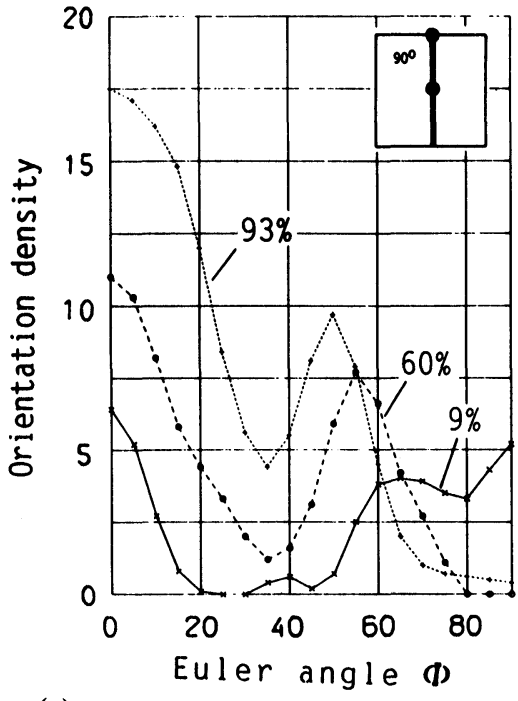

(a)

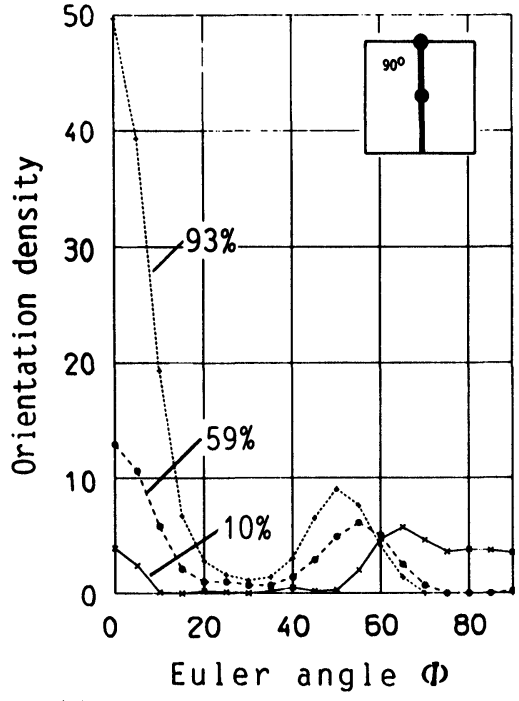

(b)

Figure 15 Orientation density along the section $\varphi_{1}=90^{\circ}, \varphi_{2}=45^{\circ}$. (a) Two-stage rolling. (b) Multi-stage rolling. 


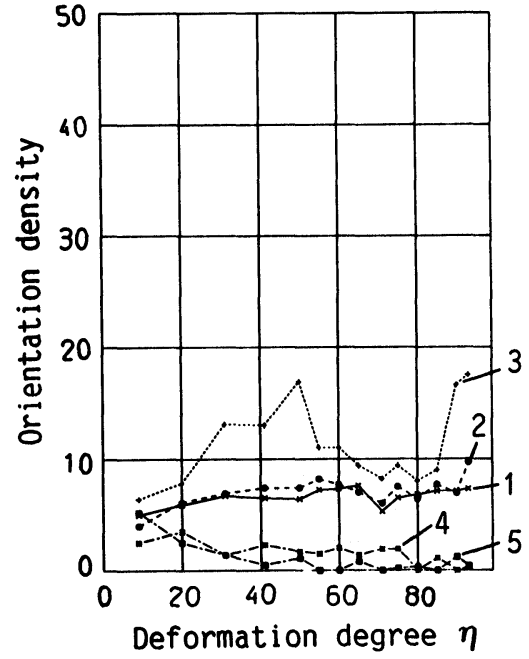

(a)

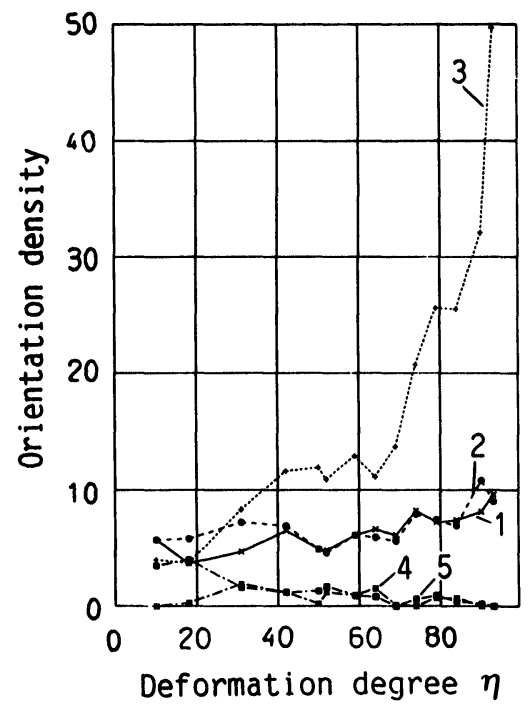

(b)

Figure 16 Orientation densities of the main ideal orientations as a function of the degree of deformation. (a) Two-stage rolling. (b) Multi-stage rolling $1=A \sim\{111\}\langle 110\rangle, 2=B \sim\{111\}\langle 112\rangle$, $3=\{001\}\langle 110\rangle, 4=\{100\}\langle 001\rangle, 5=\{110\}\langle 001\rangle$.

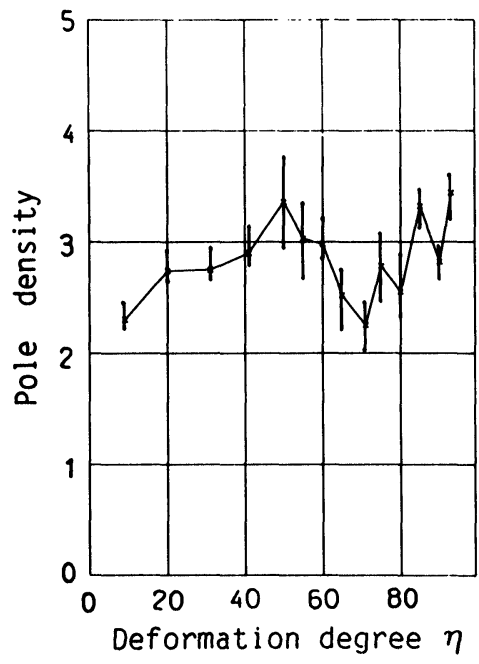

(a)

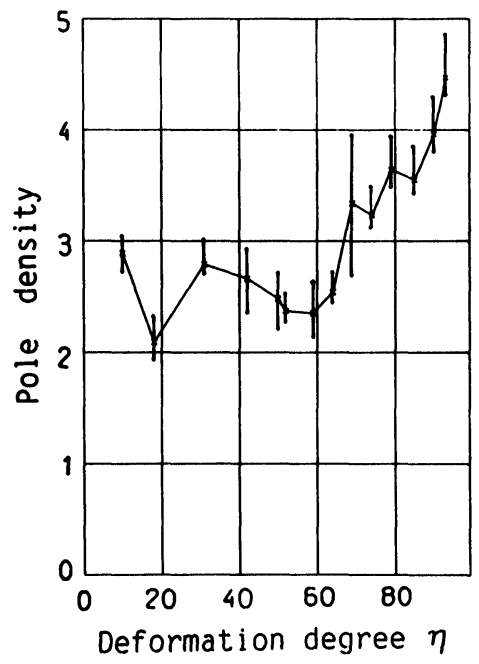

(b)

Figure 17 Pole density of the peak-points of the (110)-pole figure as a function of the degree of deformation. The error margin corresponds to the scatter of the values of equivalent points. (a) Two-stage rolling. (b) Multi-stage rolling. 
deformation degrees of the two-stage mode. The orientation densities in the main ideal orientations given in Figure 6 together with the spread ranges between them are shown in Figures 13-15. These figures contain the ODF sections at $\left(\varphi_{1}=0^{\circ}, \varphi_{2}=45^{\circ}\right),\left(\varphi_{1}=0^{\circ}, \phi=0^{\circ}\right)$ and $\left(\varphi_{1}=90^{\circ}, \varphi_{2}=45^{\circ}\right)$ respectively. The development of the cross-rolling textures of the two deformation modes is finally summarized in Figure 16 which gives the orientation densities at the main points indicated in Figure 6. In both modes, the orientation density of the tube orientations $A$ and $B$ (and in between them) increases continuously. The "rotated cube orientation" (001)[110] increases very strongly in the multistage mode. It also increases (with an intermediate minimum) in the two-stage mode. All other orientations decrease in both modes.

The development of the peak intensities in the five main orientations of Figure 6 as given in Figure 16 may be influenced by the series truncation error, especially for the higher deformation degrees. Hence, the development of the pole densities in the pole figures was also plotted as a function of the deformation degree as is shown in Figure 17. In this figure the density values of the four symmetrically equivalent pole figure points are given as error margins about the mean values. Hence, this figure also gives an estimation of the experimental accuracy. The error margins in Figure 17 may give an estimation of the accuracy of the ODF based on these pole figure values. It is to be noted that the maximum orientation density in the pole figures is much smaller than that in the ODF.

\section{DISCUSSION}

In order to end up with nearly the same thickness after deformation degrees between $10 \%$ and $93 \%$, different initial sheet thicknesses had to be used. This could not only be achieved by machining. Hence, for the smaller starting thicknesses, i.e. the lower deformation degrees, the thickness of the as received material was reduced additionally by rolling followed by recrystallization. This pre-treatment gives rise to different initial states of the material before crossrolling. This refers to the grain structures of the materials as well as to their textures. The grains of the only machined state A4 were equiaxed and the starting texture consisted mainly of a $\{111\}$-fibre. The grains of the additionally cold rolled and recrystallized materials became increasingly elongated in rolling direction and the textures developed additionally a $\{001\}\langle 110\rangle$ orientation. Also the maximum orientation density increased up to $5.74 \times$ random as is seen in Figure 2. These differences in the initial state may be considered as a disadvantage. On the other hand, however, they also provide a means of estimating the influence of the initial state on the final cross-rolling textures.

Cross-rolling was carried out in two different ways, i.e. the truly tetragonal "multi-stage" rolling consisting of a large number of small steps with a rotation of $90^{\circ}$ after each step and "two-stage" rolling. In this latter case, the rolling direction was turned by $90^{\circ}$ only once after half of the total reduction in thickness. Up to about $50 \%$ deformation this corresponds approximately to "balanced" two-stage rolling i.e. the partial rolling degrees in longitudinal and transverse direction are approximately equal as is to be seen in Figure 3 . At higher deformation degrees the second partial deformation degree $\eta_{2}$ becomes increasingly stronger than the first one, $\eta_{1}$. Hence, the resulting textures are 
dominated by $\eta_{2}$, i.e. they become more similar to straight-rolling textures corresponding to the second rolling direction. This is seen in Figure 5e which resembles a straight-rolling texture of iron but with $\varphi_{1}$ rotated through $90^{\circ}$.

The texture of straight-rolled iron was described with two complementary models, i.e.

a) by three ideal orientations $\{001\}\langle 110\rangle,\{211\}\langle 110\rangle,\{111\}\langle 112\rangle$ with spread ranges between them.

b) by two fibre axis components $\langle 110\rangle \| \mathrm{RD}$ and $\langle 111\rangle \| \mathrm{ND}$ with increased intensities at their intersection points.

In the first model, the orientation $\{001\}\langle 110\rangle$ has a four-fold symmetry axis $\langle 001\rangle \| N D$. Hence, this orientation should equally be favoured by rolling in longitudinal and transverse direction. This is corroborated by the results represented in Figure 16. The orientation density in this orientation increases extremely at the higher rolling degrees in two-stage rolling but particularly in multi-stage rolling.

Of all the other orientations occurring in the straight-rolling texture of iron, no one possesses this same four-fold symmetry axis. Hence, all the other orientations must be assumed to oscillate from pass to pass in multi-stage rolling. This is also corroborated by Figure 16a, showing that all other orientations reach maximum densities which are much smaller than the density of $\{001\}\langle 110\rangle$.

In the second model used for the description of the straight-rolling texture of iron, the $\langle 111\rangle \| \mathrm{ND}$-fibre component as a whole has axial symmetry with respect to normal direction. This symmetry includes the four-fold axis of tetragonal symmetry. Hence, it must be assumed that this fibre component is a "dynamical" end-orientation for cross-rolling. By this, it is meant that the individual orientations belonging to this fibre are not really stable to cross-rolling. Rather the crystals in these orientations migrate along or around the skeleton line of the $\langle 111\rangle \|$ ND-fibre. Such a behaviour is corroborated by the steady increase of the orientation densities in the two main tube orientations $\{111\}\langle 110\rangle$ and $\{111\}\langle 112\rangle$ as is seen in Figure 16 and the constant orientation density along the skeleton line of multi-stage rolling as is shown in Figure 12b.

The minor orientation $\{110\}\langle 001\rangle$ develops only at low deformation degrees and decreases in intensity with higher deformation degrees. For the lower deformation degrees the initial state $\mathrm{Al}$ was used which did not contain this orientation. Hence, the initial evolution and subsequent decrease of this orientation must be assumed to be relevant. There was also a small amount of cube texture $\{001\}\langle 100\rangle$ at some of the intermediate deformation degrees of multi-stage rolling. This orientation must, however, be assumed to be in the order of the error of the ODF.

Texture development after two-stage rolling is very similar to that of multistage rolling up to approximately $50 \%$ of deformation. Up to this degree two-stage rolling can be considered as nearly "balanced" in the two rolling directions. Slight differences in the orientation density values between the two rolling modes up to $50 \%$ must be assumed to be within the uncertainty of the ODF.

Differences between the two rolling modes evolve, however, above $50 \%$. At these degrees, the second stage, i.e. rolling in transverse direction predominates. The most obvious feature in this range is the decrease of intensity of the 
orientation $\{001\}\langle 110\rangle$. Only at the highest deformation degrees the intensity in this orientation increases again. This effect is not easily understood. In principle, the orientation density in a stable orientation is the result of flow of neighbouring orientations into the stable ones. Principally, both modes of cross-rolling are characterized by "broken" deformation paths. In multi-stage rolling the deformation tensor is changed after each rolling pass. Since these passes were rather small, every two may be combined to one pass which then has tetragonal symmetry. These combined passes are the same throughout the whole rolling process. Hence, multi-stage rolling can be described by a straight deformation path which corresponds to the tetragonal symmetry. Two-stage rolling, on the other hand, corresponds to a "broken" path consisting of two straight paths, each of which corresponds to orthorhombic deformation symmetry. Although the orientation $\{001\}\langle 110\}$ must be assumed to be stable according to both deformation modes, the flow in the vicinity of this orientation must be different such that different regions of the starting texture will finally flow into this orientation. A quantitative understanding of this behaviour cannot be expected without quantitative model calculations using flow fields in the Euler space. Such calculations are presently being carried out and will be reported elsewhere.

A minor difference between the two rolling modes is furthermore the behaviour of the cube orientation. In two-stage rolling, the amount of cube orientation was found in the same order of magnitude as that of the Gossorientation $\{011\}\langle 100\rangle$. In the initial state, the cube orientation was not present to any considerable extent. Hence, also the cube orientation must be assumed to be an intermediate orientation in the case of two-stage rolling.

\section{SUMMARY AND CONCLUSIONS}

Cross-rolling in iron was carried out in two different modes, i.e. multi-stage rolling with a rotation of $90^{\circ}$ after each rolling pass and two-stage rolling consisting of longitudinal rolling to about half of the reduction in thickness followed by transverse rolling to the final thickness. Multi-stage rolling virtually follows a straight deformation path with tetragonal symmetry whereas two-stage rolling corresponds to a "broken"deformation path, the two parts of which have only orthorhombic symmetry. Since the addition of crystal rotations is not commutative, this broken path as a whole has only orthorhombic symmetry. On the other hand, as long as the two partial deformation degrees of two-stage rolling are nearly equal, the resulting textures are similar. Truly tetragonal multi-stage rolling gives rise to an extremely high density in the orientation $\{001\}\langle 110\rangle$ and a nearly ideal fibre axis component $\langle 111\rangle \|$ ND. All other orientations decrease in intensity with increasing deformation degree. The texture after two stage rolling develops in a similar way up to about $50 \%$ deformation. After that the intensity in the predominant orientation $\{001\}\langle 110\rangle$ decreases and increases again at the very highest deformation degrees.

\section{ACKNOWLEDGEMENT}

The authors wish to express their thanks to Deutsche Forschungsgemeinschaft for financial support of the work. 


\section{References}

Haeßner, F. and Weik, H. (1956). Untersuchung der Walz- und Rekristallisationstexturen von Karbonyleisen. Arch. für das Eisenhüttenwesen 27, 153-160.

Kern, R., Lee, H. P. and Bunge, H. J. (1986). The Rolling Texture of Iron of different purities. Steel Research 57, 563-571.

Öztürk, T. (1988). Deformation and Rekristallization Textures in Cross-rolled Sheets of Copper and $\alpha$-Brass. Scripta Met 22, 1611-1616.

Wassermann, G. and Grewen, J. (1969). Texturen metallischer Werkstoffe. Springer Verlag Berlin.

Yeung, W. Y. and Duggan, B. J. (1986). Texture and Structure Development in Cross-rolled $\alpha$-Brass. Acta Met 34, 653-660. 